\title{
Correction to: A Post-Nationalist History of Television in Ireland
}

\section{Correction to:}

E. Brennan, A Post-Nationalist History of Television in Ireland, https://doi.org/10.1007/978-3-319-96860-5

Post-publication corrections have been incorporated throughout this book. 